\title{
miR-410-3p Suppresses Cytokine Release from Fibroblast-Like Synoviocytes by Regulating NF-KB Signaling in Rheumatoid Arthritis
}

\author{
YueJiao Wang, ${ }^{1}$ NeiLi Xu, ${ }^{1}$ Shuai Zhao, ${ }^{1}$ Ting Jiao, ${ }^{1}$ WenYi Fu, ${ }^{1}$ LiLi Yang, ${ }^{1}$ and Ning Zhang ${ }^{1,2}$
}

\begin{abstract}
R-410-3p acts as an oncogene or a tumor suppressor in some malignancies. However, its role in rheumatoid arthritis (RA) is unknown. The study was conducted to investigate the effect of miR-410-3p on the pathogenesis of RA. Real-time RT-PCR was used to determine the mRNA levels of miR-410-3p in synovial tissues and fibroblast-like synoviocytes (FLSs). An ELISA was performed to examine the production levels of tumor necrosis factor (TNF)- $\alpha$, interleukin (IL)-1 $\beta$, IL-6, and matrix metalloproteinase (MMP)-9. Western blotting was conducted to determine the protein levels of IкB- $\alpha$, p-IKB $\alpha$, p65, and p-p65. Nuclear factor (NF)$\mathrm{KB}$ activation and nuclear translocation assays were performed to confirm the activation of NF$\mathrm{kB}$. We found that the expression level of miR-410-3p was downregulated in synovial tissues and FLSs from RA. Overexpression of miR-410-3p significantly reduced the secretion of TNF- $\alpha$, IL$1 \beta$, IL-6, and MMP-9 in human RA fibroblast-like synoviocytes (HFLS-RA); whereas miR-410$3 p$ inhibition increased the expression levels of these cytokines. Furthermore, miR-410-3p suppresses the activation of NF- $\mathrm{kB}$ signaling pathway. Moreover, NF- $\mathrm{kB}$ inhibitor restored the elevation of TNF- $\alpha$, IL-1 $\beta$, IL-6, and MMP-9 induced by miR-410-3p inhibition. Our results demonstrate that miR-410-3p acts an inflammatory suppressor in the pathogenesis of RA by regulating the NF- $\mathrm{kB}$ signaling pathway. These data suggest a novel function of miR-410-3p and provide insight into the complex mechanisms involved in RA.
\end{abstract}

KEY WORDS: rheumatoid arthritis; miR-410-3p; fibroblast-like synoviocytes; cytokines; NF-kB signaling.

\section{INTRODUCTION}

Rheumatoid arthritis (RA) is a chronic autoimmune inflammatory disease that causes progressive articular damage, functional loss, and comorbidity. RA affects approximately $1 \%$ of the population, can develop at any age, and is more prevalent in women than in men [1]. An improved understanding of RA pathogenesis has led to several

\footnotetext{
${ }^{1}$ The Department of Rheumatology, Shengjing Hospital of China Medical University, Shenyang, Liaoning, China

${ }^{2}$ To whom correspondence should be addressed at The Department of Rheumatology, Shengjing Hospital of China Medical University, Shenyang, Liaoning, China. E-mail: nzhang55@cmu.edu.cn
}

therapeutic options; however, additional studies are needed to avoid side effects and improve the quality of life of RA patients [2]. Current treatment strategies involve traditional disease-modifying anti-rheumatic drugs and novel biologic agents targeting T cells, B cells, pro-inflammatory cytokines including tumor necrosis factor (TNF) or interleukin (IL)-1, and tyrosine kinase activity both markedly improve the clinical outcomes of RA patients [2, 3]. However, at least $30 \%$ of RA patients show poor responses to the available therapies, suggesting that novel mediators are needed for targeting of other disease-specific pathways or cell lineages.

Fibroblast-like synoviocytes (FLSs), also known as synovial fibroblasts, are a quite unique cell type that distinguishes RA from other inflammatory conditions of the 
joints and are key effector cells in the pathogenesis of RA [4]. FLSs from RA patients exhibit a unique progressive phenotype that increases invasiveness into the extracellular matrix and further exacerbates joint damage. This invasiveness of FLSs is considered equivalent to that of tumor cells [5]. Numerous studies showed that FLSs participate in cell inflammation by secreting cytokines and chemokines, particularly TNF- $\alpha$, IL- $1 \beta$, and IL- 6 , and result in joint tissue damage in RA [6]. Furthermore, inflammatory cytokines secreted by FLSs increase the total number of FLSs and promote the development of systemic inflammation, such as chronic anemia and cardiovascular disease [7]. Therefore, understanding the molecular mechanism of dysregulation of cytokines expression in RA patients, particularly in FLSs, may provide novel treatment strategies.

MicroRNAs (miRNAs) are endogenous small noncoding RNA molecules that modulate the expression of multiple protein-encoding genes at the post-transcriptional level [8]. Accumulating evidence has shown that these small RNA molecules play important roles in various pathological conditions, including rheumatic and other autoimmune diseases $[9,10]$. Several studies suggested that miRNAs are involved in controlling the inflammatory response of immune and non-immune cells. For instance, miR-124 mediates the anti-inflammatory action by suppressing production of the pro-inflammatory cytokines IL-6 and TNF- $\alpha$ [11]. Lucas Philippe et al. observed dysregulated expression of miR-20a decreased IL-6 and CXCL10 released by RA FLSs and IL- $1 \beta$ and TNF- $\alpha$ by activated THP-1 cells [12]. Recent studies suggested that miR-410-3p is involved in inflammation, angiogenesis, and tumorigenesis [13-15]. Furthermore, as a key regulatory factor in the pathogenesis of systemic lupus erythematosus (SLE), miR-410-3p was found to be decreased in T cells of SLE patients and regulated the expression of IL10 [16]. However, no studies have examined miR-410-3p expression and its roles in RA.

In this study, we explored the expression of miR-410$3 p$ in FLSs and synovial tissues of RA patients, its role in inflammatory cytokine secretion in RA-FLSs, and the underlying mechanisms.

\section{METHODS}

\section{Cell Lines and Tissues}

Human RA fibroblast-like synoviocytes (HFLS-RA) and human normal fibroblast-like synoviocytes (HFLS) were purchased from Jennio Biotech Co., Ltd.
(Guangzhou, China). HFLS-RA were cultured in DMEM (Gibco, Life Sciences, MD), and HFLS were cultured in MEM (Gibco), supplemented with $10 \%$ heat-inactivated fetal bovine serum (FBS) (Gibco), penicillin (final concentration, $100 \mathrm{U} / \mathrm{ml}$ ), and streptomycin (final concentration, $0.1 \mathrm{mg} / \mathrm{ml}$ ) (all from Hyclone, Logan, UT), in a humidified atmosphere of $5 \% \mathrm{CO}_{2}$ and $95 \%$ air at $37{ }^{\circ} \mathrm{C}$. Cells were passaged every $4-5$ days. Cells from the 3 rd- 8 th passages were used for the following experimental procedures.

Synovial tissues were obtained from RA patients at the time of arthroscopic biopsy or total joint replacement from the Department of Orthopaedics in the Shengjing Hospital of China Medical University. All five RA patients met the 1987 American College of Rheumatology (ACR) criteria for the classification of RA [17]. Four control synovial tissue samples were obtained from patients with joint trauma during routine arthroscopy or open joint surgery for diagnostic and therapeutic procedures. All patients were informed of the purpose of the study and gave written consent. The study was approved by the Ethics Committee of the Shengjing Hospital of China Medical University.

\section{Transfection Experiments}

The experimental setup consisted of five groups: the blank group, miR-410-3p mimics group (transfected with miR-410-3p mimics), mimics-negative control (transfected with miR-410-3p mimics-negative control sequence), miR-410-3p inhibitor group (transfected with miR-410$3 p$ inhibitor), and inhibitor-negative control (transfected with miR-410-3p inhibitor-negative control sequence). miR-410-3p-related sequences were synthesized by GenePharma (Shanghai, China). HFLS-RA cells were seeded into six-well plate, transfected using Lipofectamine $^{\mathrm{TM}} 3000$ Reagent (Invitrogen, USA). The respective miR-410-3p sequences and Lipofectamine 3000 were mixed separately with Opti-MEM (Invitrogen) and then incubated together at room temperature for 10 min under serum-free conditions to form transfection complexes. The HFLS-RA was washed twice with PBS and the corresponding transfection complexes were added to each well. Complete DMEM media was replaced $6 \mathrm{~h}$ later. The transfection efficiency was verified by fluorescence microscopy at $24 \mathrm{~h}$, and cells at $48 \mathrm{~h}$ and $72 \mathrm{~h}$ post-transfection were used for further analysis.

\section{miR-410-3p Quantification}

HFLS-RA cells were first transfected with miR-410$3 \mathrm{p}$ mimics, inhibitor, or NC for $48 \mathrm{~h}$. Quantification of miR-410-3p was performed with real-time RT-PCR as 
previously described with modifications [18]. In brief, total RNA containing small-size RNA was isolated or extracted from synovial tissues and cells with RNAiso Plus reagent (Takara, Japan). Total RNA (3 $\mu \mathrm{g})$ was reversely transcribed to cDNA using a Mir-XTM miRNA First-Strand Synthesis kit (Takara). Next, the cDNA was amplified with a SYBR ${ }^{\circledR}$ Premix Ex Taq ${ }^{\text {TM }}$ II (Tli RNaseH Plus) kit (Takara). U6 was used as an internal control and the relative expression of miR-410-3p was calculated using the 2- $\Delta \Delta C T$ method. The PCR primers were commercially obtained from Sangon Biotech (Shanghai, China).

\section{Western Blot}

HFLS-RA cells were first transfected with miR-410$3 p$ mimics, inhibitor, or NC for $72 \mathrm{~h}$, and then were harvested and lysed using RIPA buffer (Beyotime Biotechnology, Shanghai, China). The protein concentration was determined with a BCA kit (Beyotime Biotechnology) according to the protocol. Equal amounts of protein corresponding to approximately $30 \mu \mathrm{g}$ were subjected to SDSPAGE. Then, proteins were transferred to polyvinylidene difluoride (PVDF) membranes and incubated with $5 \%$ BSA for $2 \mathrm{~h}$. Diluted primary antibodies (IкB- $\alpha, \mathrm{p}-\mathrm{I} \kappa \mathrm{B} \alpha$, p65, and p-p65; Cell Signaling Technology, USA; GAPDH and $\beta$-actin; ZSGB-BIO, China) were added into the membranes, and the mixture was incubated at $4{ }^{\circ} \mathrm{C}$ overnight. The membranes were washed with Trisbuffered saline Tween-20 (TBST). Horseradish peroxidase-labeled secondary antibodies (ZSGB-BIO) were added and the mixture was incubated at room temperature for $2 \mathrm{~h}$. The membrane was then washed with TBST three times for $10 \mathrm{~min}$ once. The bands were visualized by enhanced chemiluminescence (ECL) according to the instructions (Millipore). The protein bands were quantitatively analyzed by using Image J software, normalized to GAPDH or $\beta$-actin.

\section{Assessment of NF-кB Nuclear Translocation}

NF-KB activation and nuclear translocation assay was performed according to the reagent manufacturer's instructions (Beyotime Biotechnology). Briefly, $24 \mathrm{~h}$ after being plated in six-well plate, HFLS-RA cells were transfected with miR-410-3p mimics, inhibitor, or NC for $72 \mathrm{~h}$, then fixed and blocked at room temperature. After incubation with rabbit anti-p65 NF- $\mathrm{kB}$ antibody overnight at $4{ }^{\circ} \mathrm{C}$, cells were added with fluorescent secondary antibody. Eventually, the nuclear was dyed with DAPI (Beyotime Biotechnology). The fluorescence microscope was used to take pictures.

\section{Enzyme-Linked Immunosorbent Assay}

HFLS-RA cells were seeded into 24 well plates and transfected with miR-410-3p mimics, inhibitor, or NC for $48 \mathrm{~h}$, with or without BAY 11-7082 treatment (Beyotime Biotechnology). The concentrations of TNF- $\alpha$, IL-1 $\beta$, IL6 , and MMP-9 in the cell culture supernatants were determined by a sandwich enzyme-linked immunosorbent assay (ELISA) (R\&D Systems, Minneapolis, MN) according to the manufacturer's instructions. A standard curve was performed for each plate and used to calculate the absolute concentrations of the indicated cytokines.

\section{Statistical Analysis}

All data were expressed as the mean \pm standard deviation from at least three independent experiments and normally distributed. The differences between two groups were analyzed using the two-tailed Student $t$ test. For the differences among three groups, one-way ANOVA followed by Tukey's multiple comparisons test was used. $P$ values below 0.05 was considered as statistically significant (ns, non-significant, $* p<0.05$ ). All calculations were performed using the GraphPad Prism version 7.00 (GraphPad Software, Inc., San Diego, CA, USA).

\section{RESULTS}

\section{miR-410-3p Is Reduced in Synovial Tissues and Fibroblast-Like Synoviocytes in RA Patients}

We first tested the miR-410-3p expression in synovial tissues in RA patients. Synovial tissue samples were isolated from both non-RA controls and RA patients, and miR-410-3p expression was quantified. As shown in Fig. 1, the miR-410-3p expression was significantly decreased in the synovial tissues from RA patients $(p<0.05)$. Next, we compared the miR-410-3p level between two synovial cell lines, and the miR-410-3p level was lower in HFLS-RA $(p<0.001)$, indicating that aberrant miR410-3p expression is associated with the pathogenesis of RA.

\section{Effect of miR-410-3p Expression on HFLS-RA Cyto- kine Release}

To measure the effect of miR-410-3p levels, miR410-3p mimics and inhibitor were used to manipulate the expression level of miR-410-3p in HFLS-RA. Total RNA was extracted at $48 \mathrm{~h}$ after transfection to detect the relative expression of miR-410-3p. As shown in Fig. 2a, miR-410- 
a

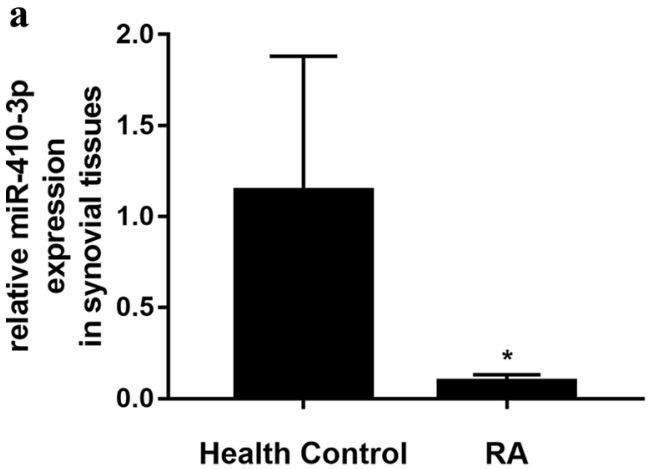

b



Fig. 1. miR-410-3p is reduced in synovial tissues and FLSs in RA patients. Synovial tissue samples were isolated from non-RA controls and RA patients, and miR-410-3p level was determined by real-time RT-PCR. All samples were performed in triplicates for each condition. Data shown were mean \pm SD of three independent experiments. a Expression of miR-410-3p in synovial tissues of 4 HCs and in the 5 patients with RA. b Expression of miR-410-3p in HFLS and HFLS-RA. $* p<0.05, * * * P<0.001$, compared with control.

3 p level was reduced to approximately $20 \%$ after miR-410$3 p$ inhibitor transfection $(p<0.01)$, whereas it was markedly increased after miR-410-3p mimics transfection $(p<0.0001)$.

At $48 \mathrm{~h}$ after transfection, cytokines levels, including those of TNF- $\alpha$, IL-1 $\beta$, IL-6, and MMP-9, secreted from HFLS-RA were evaluated by ELISA. It was presented that TNF- $\alpha$, IL-1 $\beta$, IL-6, and MMP-9 levels were clearly downregulated in HFLS-RA transfected with miR-410-3p mimics $(p<0.05)$, whereas they were significantly elevated in HFLSRA transfected with miR-410-3p inhibitor $(p<0.05)$ (Fig. $2 \mathrm{~b}-\mathrm{e})$. These results indicate that miR-410-3p suppressed the secretion of inflammatory cytokines in HFLS-RA.

\section{Effect of miR-410-3p in HFLS-RA on the NF- $\kappa B$ Sig- naling Pathway}

To explore the potential mechanism underlying miR410-3p-suppressed cytokine release in HFLS-RA, we conducted western blotting to test whether miR-410-3p regulates the NF-KB signaling pathway. The results showed that compared to the $\mathrm{NC}$ and blank groups, $\mathrm{p}-\mathrm{I} \mathrm{KB} \alpha$ and p-p65 levels were significantly increased in the HFLS-RA transfected with miR-410-3p inhibitor (all $p<0.05$ ) (Fig. 3a, b, d). In contrast, the total IkB- $\alpha$ and p65 levels showed no difference among the five groups (Fig. 3c, e). Furthermore, a nuclear translocation assay was performed to confirm the activation of NF- $\mathrm{KB}$. The results showed that miR-410-3p upregulation suppressed the activation of NF- $k B$ in HFLS-RA, while miR-410-3p inhibition activated NF-KB signaling (Fig. 4). These results indicate that in HFLS-RA, miR-410-3p overexpression suppressed the NF-KB signaling pathway.

\section{miR-410-3p Suppresses Cytokine Release in HFLS-RA} by the NF-KB Signaling Pathway

Given the effect of miR-410-3p on the NF-kB signaling pathway, we further explored whether miR-410-3p suppressed cytokine release in HFLS-RA by regulating the NF- $\mathrm{KB}$ signaling pathway. To evaluate the involvement of NF- $\mathrm{KB}$ mediated by miR-410-3p, we pretreated the HFLS-RA with BAY 11-7082, an inhibitor of NF-KB nuclear translocation. As indicated in Fig. 5a-c, in the presence of BAY 11-7082, the levels of the p-p65 was significantly reduced $(p<0.0001)$, while total p65 levels showed no difference. Additionally, as shown in Fig. 5d-g, elevations of inflammatory cytokines, including TNF- $\alpha$, IL-1 $\beta$, IL-6, and MMP-9, in HFLS-RA induced by miR410-3p inhibition was rescued by BAY 11-7082 (all $p<0.001$ ), indicating that miR-410-3p suppressed cytokine release in HFLS-RA by regulating the NF- $\mathrm{KB}$ signaling pathway.

\section{DISCUSSION}

RA is a systemic chronic inflammatory disease mainly characterized by hyperplastic synovial pannus tissue, which mediates the destruction of cartilage and bone [19]. FLSs are key components of this invasive synovium and play a major role in the initiation and perpetuation of destructive joint inflammation. As primary promoters of inflammation, FLSs from RA patients also display unique aggressive features. These FLS cells contribute to the inflammatory microenvironment and recruit and activate immune cells to the 
$\mathbf{a}$

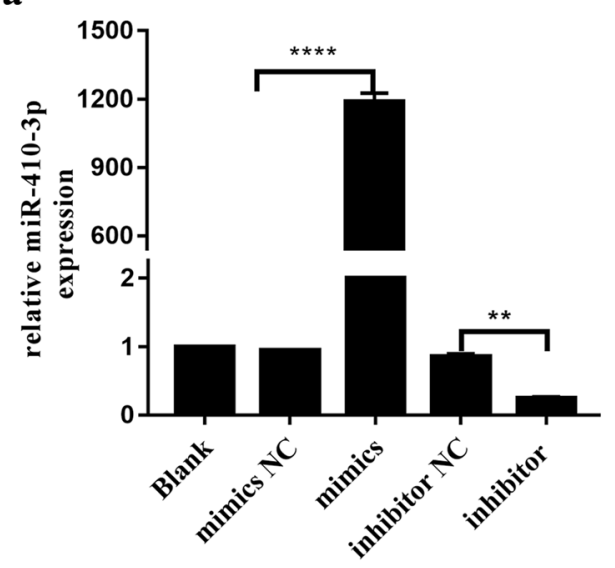

c

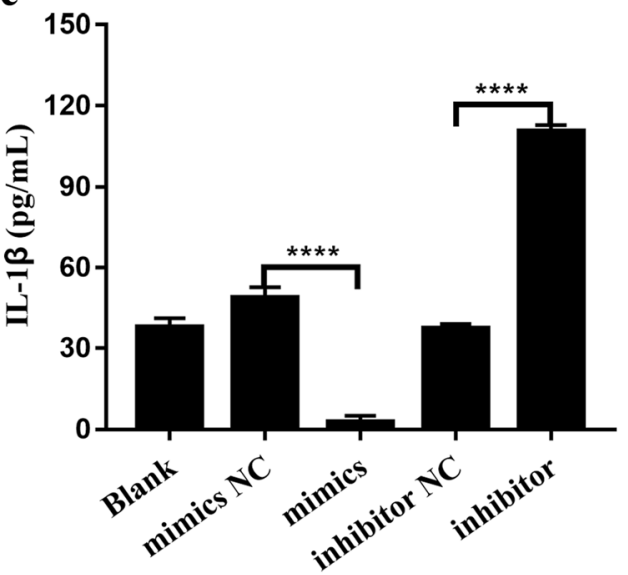

e

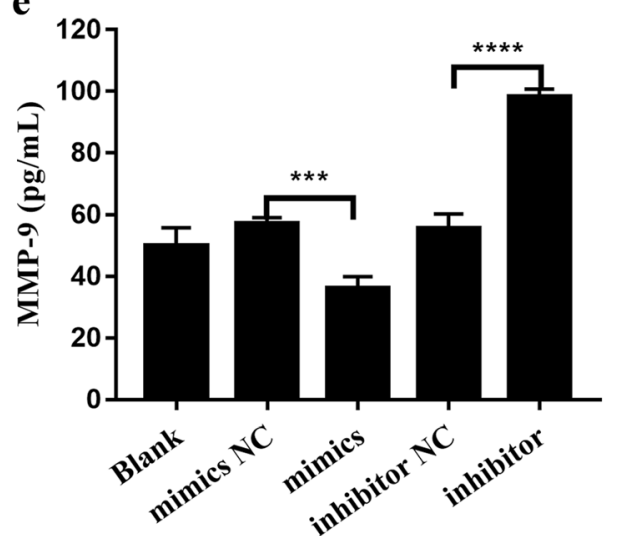

b

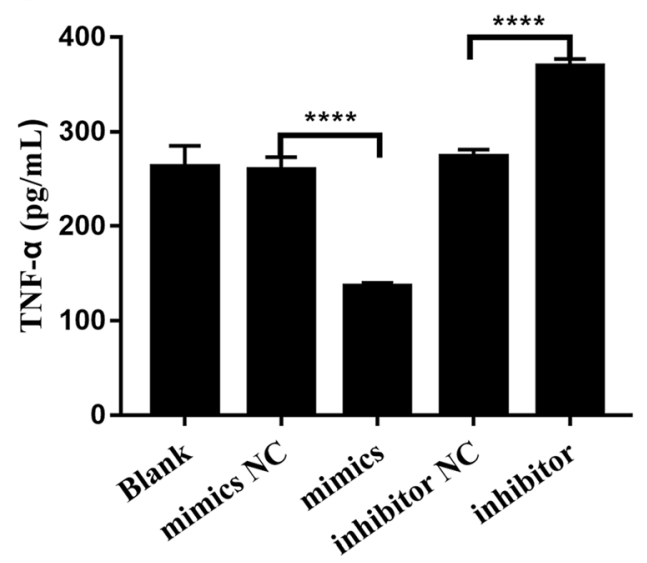

d

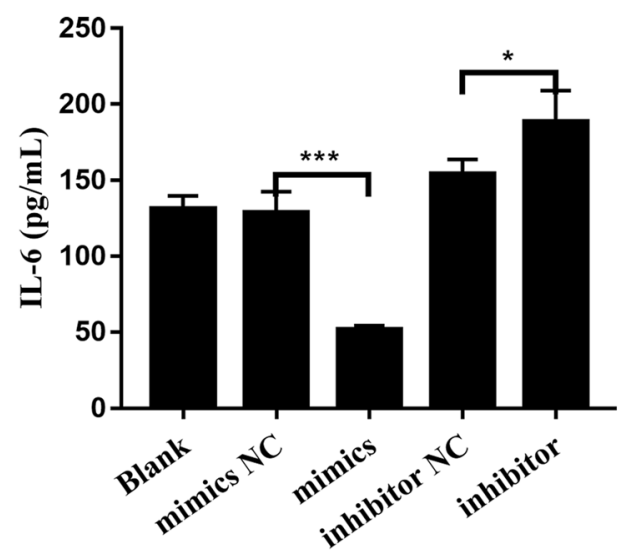

Fig. 2. Effect of miR-410-3p expression on HFLS-RA cytokine release. miR-410-3p mimics and inhibitor were designed and transfected into HFLS-RA followed by measuring the expression of miR-410-3p by real-time RT-PCR and the secretion of TNF- $\alpha$, IL-1 $\beta$, IL-6, and MMP-9 by ELISA. a Expression levels of miR-410-3p in HFLS-RA after transfection. b-e Relative expression levels of TNF- $\alpha$, IL-1 $\beta$, IL-6, and MMP-9 in HFLS-RA after transfection. $* p<0.05, * * p<0.01, * * * p<0.001, * * * * p<0.0001$, compared with control. 
a

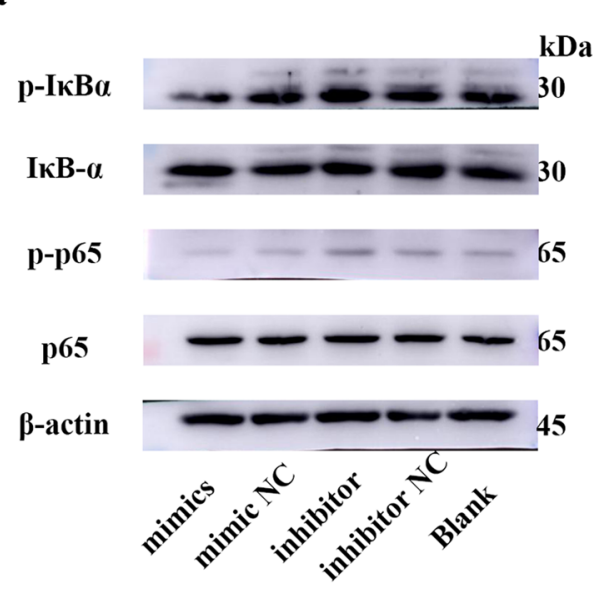

c

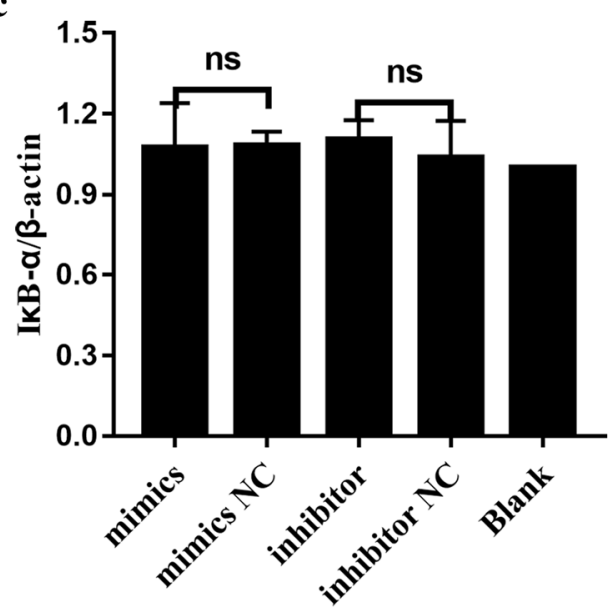

e

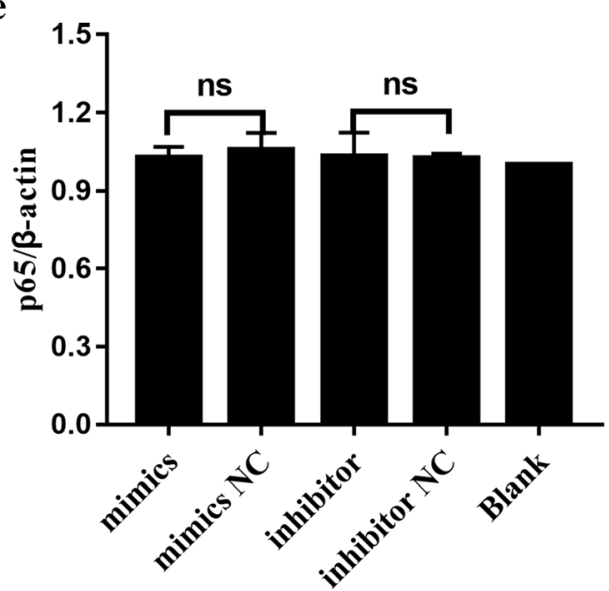

b

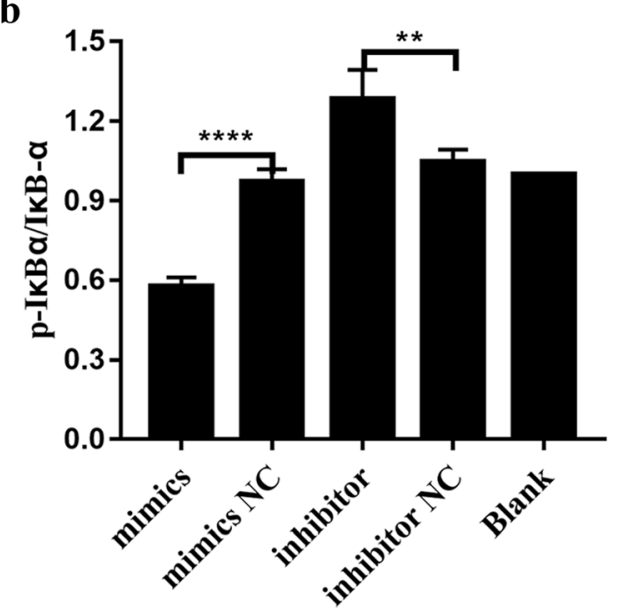

d

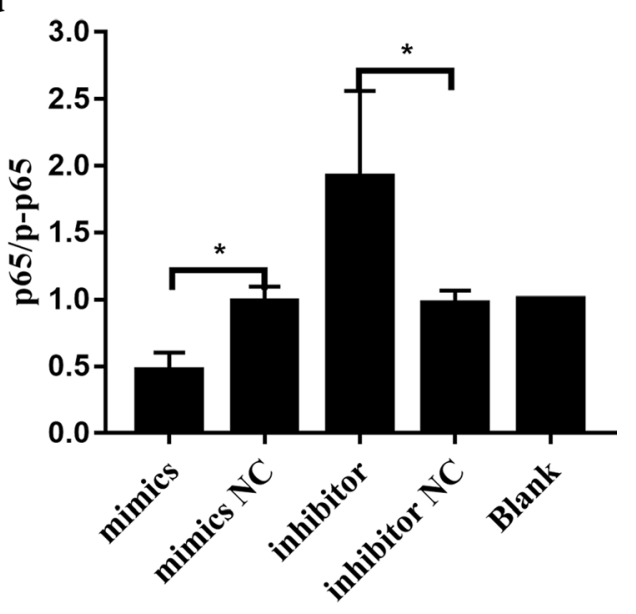

Fig. 3. Effect of miR-410-3p in HFLS-RA on NF-kB signaling pathway. miR-410-3p mimics and inhibitor were transfected into HFLS-RA followed by

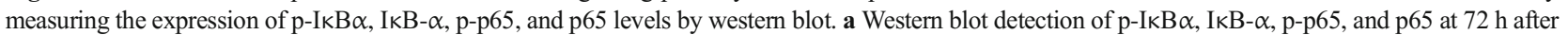
miR-410-3p transfection. b-e Histograms showing the expression of p-IкB $\alpha$, IкB- $\alpha$, p-p65, and p65 levels detected by western blot. * $p<0.05$, ** $p<0.01$, $* * * * p<0.0001$, compared with control. 
p65

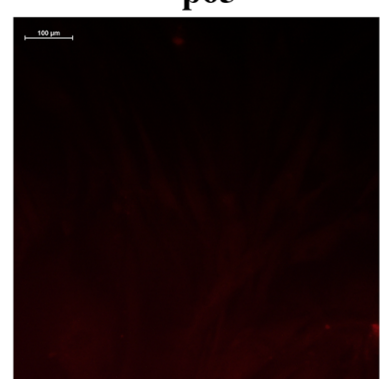

mimics

mimics NC
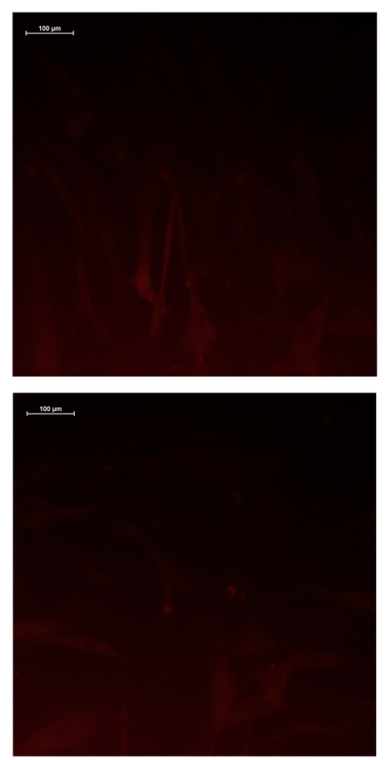

inhibitor

\section{inhibitor NC}


Merge
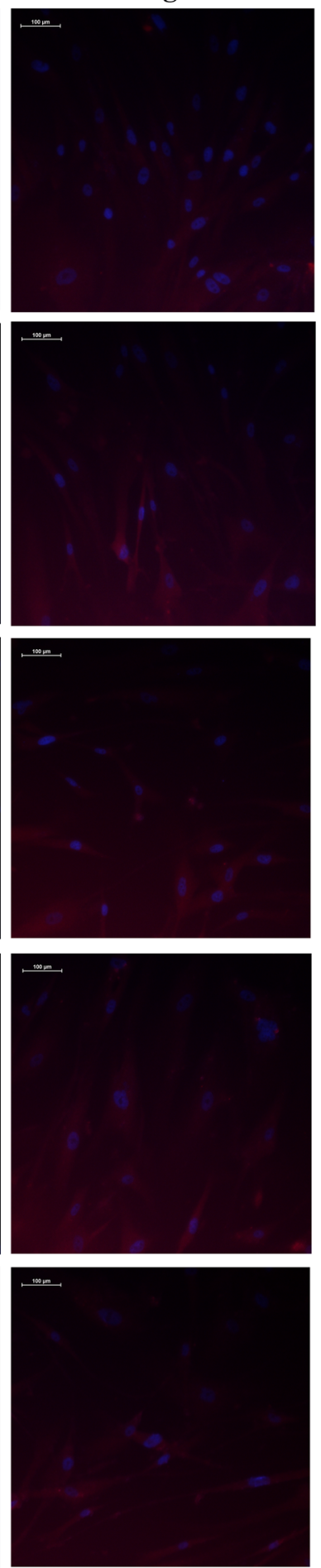

Fig. 4. Effect of miR-410-3p on NF-kB activation. HFLS-RA were seeded in a 6-well culture plate, and subsequently treated with miR-410-3p mimics, inhibitor, or NC for $72 \mathrm{~h}$. After treatment, cells were incubated with anti-p65 NF- $\mathrm{kB}$ antibody and $\mathrm{Cy} 3$ fluorescein-conjugated secondary antibody, and nuclei were stained with DAPI. The images were obtained by confocal laser microscopy and overlay. The pink fluorescence indicates location of p65 protein in nuclei. 
a

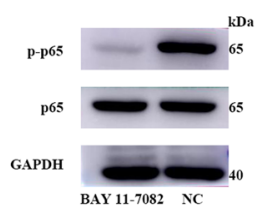

d
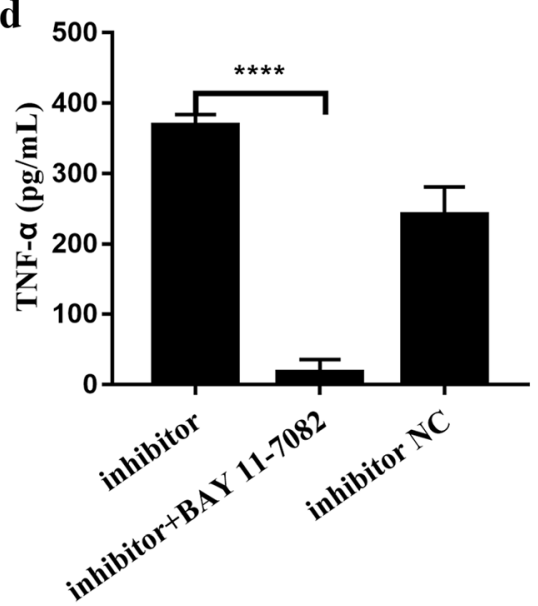

f

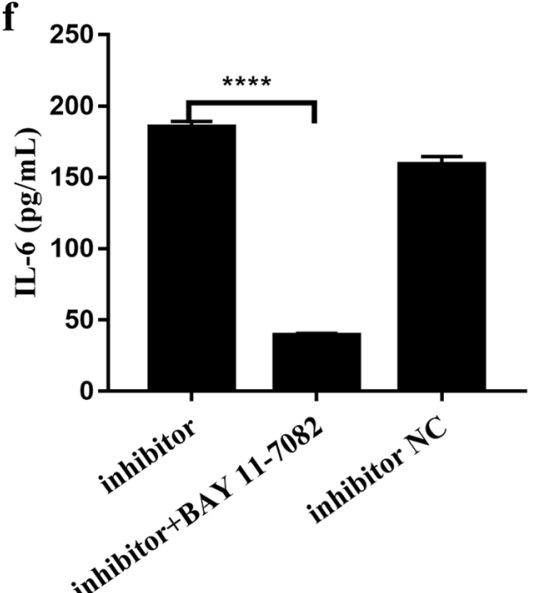



c

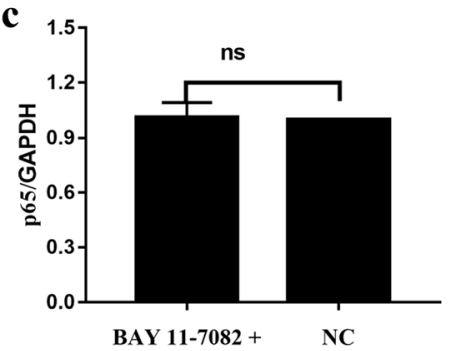

e

e 180

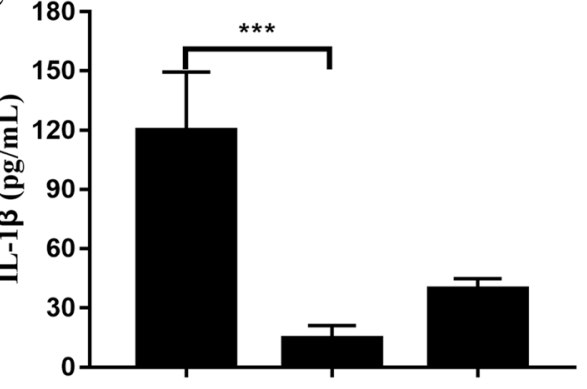

g

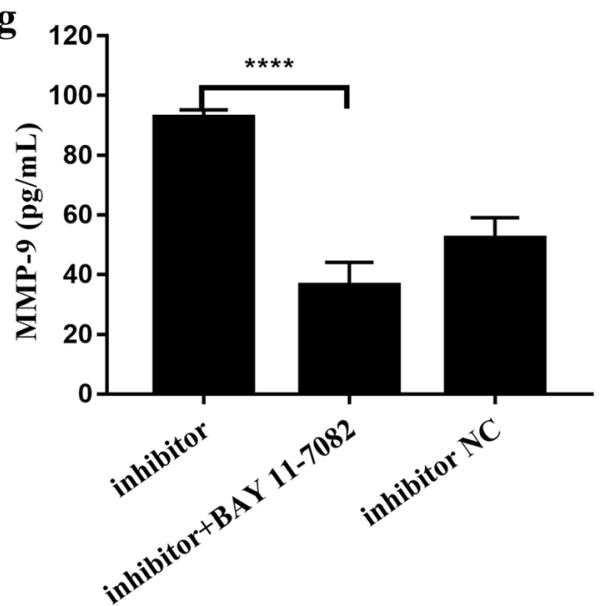

Fig. 5. miR-410-3p suppresses cytokine release in HFLS-RA by the NF- $k B$ signaling pathway. miR-410-3p inhibitor was transfected to HFLS-RA, with or without BAY 11-7082 treatment, followed by measuring the secretion of TNF- $\alpha$, IL-1 $\beta$, IL-6, and MMP-9 by ELISA. a Western blot of p-p65 and p65 after BAY 11-7082 treatment. BAY 11-7082 was dissolved in DMSO with the final concentration of $5 \mu \mathrm{M}$. Equal amount of DMSO was treated as the control group. b, $\mathbf{c}$ Histograms showing the expression of p-p65 and p65 levels detected by western blot. d-g Relative expression levels of TNF- $\alpha$, IL-1 $\beta$, IL-6, and MMP-9 in HFLS-RA after treatment. $* * * p<0.001,{ }^{*} * * * p<0.0001$, compared with control.

damaged synovium by secreting multiple proinflammatory cytokines and chemokines, particularly TNF- $\alpha$, IL-1 $\beta$, IL-6, and MMPs [20]. Elevated levels of cytokines and chemokines in the synovium modulate the expression of growth factors and result in further activation of FLSs, which undergo hyperplasia, a hallmark event in RA. Activated FLSs can undergo migration and enhance the production of pro- 
inflammatory cytokines and chemokines, particularly MMPs, which in turn mediate tissue destruction [21].

An increasing number of studies have focused on the abnormal expression of microRNAs in RA. Thus, understanding the disease-associated mechanisms of these small non-coding RNAs may provide a novel approach for the diagnosis and treatment of RA. Here, we showed that the expression of miR-410-3p was significantly lower in synovial tissues and FLSs of RA patients compared to that in healthy controls. Considering the downregulation of miR-410-3p in RA patients, this miRNA may have a critical role in RA development. Furthermore, we observed that miR-410$3 p$ suppressed the inflammatory cytokine release of RA-FLS. Since abnormally activated NF- $\mathrm{kB}$ signaling pathway was responsible for the inflammation and excess proliferation of FLSs in RA [22], we further investigated whether miR-410-3p suppresses the cytokine release of RA-FLS by regulating the NF- $\mathrm{kB}$ signaling pathway.

miR-410-3p is abnormally expressed in a variety of diseases, including cancer, inflammation, and autoimmune diseases and is involved in multiple biological processes, such as proliferation, apoptosis, differentiation of stem cells, and drug resistance [13, 16, 23-25]. Previous studies showed that miR-410-3p was highly expressed in the lung and colorectal cancer, and promoted the proliferation, invasion, and migration of cancer cells [23, 26-28]. In contrast, miR-410-3p was reported to suppress the proliferation, migration, and invasion of breast cancer and pancreatic cancer [25, 29, $30]$. These data indicate that dysregulation of miR-410$3 p$ occurs in a tissue-specific manner in different types of diseases. A recent study showed that miR-410-3p attenuated renal fibrosis in lupus nephritis mice via directly targeting IL-6 [13]. Additionally, miR-410-3p was found to be involved in the development of osteoarthritis through modulating the differentiation of mesenchymal stem cells (MSCs) into chondrocytes [31]. However, the roles of miR-410-3p in RA are unknown. Nuclear translocation and subsequent DNA binding of NF- $\mathrm{kB}$ in inflamed synovium transactivate the expression of its target genes, including TNF- $\alpha$, IL- $1 \beta$ and IL6 , and MMP-9 [32]. Therefore, NF- $\mathrm{KB}$ signaling has been extensively studied to identify its role in the pathogenesis of RA, and NF- $\mathrm{kB}$ inhibition has been examined as a therapeutic approach for the disease. Our results demonstrated that miR-410-3p suppressed NF$\mathrm{KB}$ activation in cultured HFLS-RA by suppressing the phosphorylation of I $\mathrm{KB}-\alpha$, thus resulting in low phosphorylation of $\mathrm{p} 65$. As transcription targets, elevations of TNF- $\alpha$, IL-1 $\beta$, IL- 6 , and MMP-9 induced by miR$410-3 p$ inhibition were restored by NF- $k B$ inhibitor. Together, these findings suggest that miR-410-3p is involved in cytokine release by suppressing the NF- KB signaling pathway. These results suggest that miR-410$3 p$ is an inflammatory suppressor in RA progression. However, there are still some limitations. First, the number of synovial tissue samples is relatively small. Additionally, certain animals' experiments are needed to evaluate the effect of miR-410-3p on cytokine release in vivo.

Although miR-410-3p regulates multiple biological processes in a variety of diseases, its upstream regulating mechanism is unknown. It was reported that internalized miR-410-5p, derived from pre-miR-410, degraded miR$410-3 p$ by base pairing and thus inhibited its function; blockade of the miR-410-5p upregulated the expression of miR-410-3p [14]. In contrast, CpG islands in the upstream regions of miR-410-3p were methylated; however, the expression level of miR-410-3p was not regulated by a DNA methylation inhibitor [33]. Additionally, some transcription factors were found to regulate the expression of miR-410-3p, such as myocyte enhancer factor 2 (MEF2) [34] and E2F [35]. However, little is known about the underlying mechanism of aberrant expression of miR410-3p in RA, which requires further investigation.

In conclusion, we demonstrated that miR-410-3p acts as an inflammatory suppressor in RA by suppressing the $\mathrm{NF}-\mathrm{KB}$ signaling pathway. These data suggest the potential diagnostic and therapeutic applications of miR-410-3p in RA.

\section{FUNDING}

The fund was provided by the program of the foundation in Shenyang (no. D267 and no. MF55). The corresponding author, Ning Zhang, is the author who received the funding.

\section{COMPLIANCE WITH ETHICAL STANDARDS}

Conflict of Interest. The authors declare that they have no competing interests.

Ethical Approval. The study was approved by the Ethics Committee of the Shengjing Hospital of China Medical University. 
Open Access This article is distributed under the terms of the Creative Commons Attribution 4.0 International License (http://creativecommons.org/licenses/by/ 4.0/), which permits unrestricted use, distribution, and reproduction in any medium, provided you give appropriate credit to the original author(s) and the source, provide a link to the Creative Commons license, and indicate if changes were made.

\section{REFERENCES}

1. McInnes, I.B., and G. Schett. 2017. Pathogenetic insights from the treatment of rheumatoid arthritis. Lancet 389 (10086): 2328-2337. https://doi.org/10.1016/S0140-6736(17)31472-1.

2. Burmester, G.R., and J.E. Pope. 2017. Novel treatment strategies in rheumatoid arthritis. Lancet 389 (10086): 2338-2348. https:// doi.org/10.1016/S0140-6736(17)31491-5.

3. van Vollenhoven, R.F. 2009. Treatment of rheumatoid arthritis: State of the art 2009. Nature Reviews Rheumatology 5 (10): 531-541. https://doi.org/10.1038/nrrheum.2009.182.

4. Bottini, N., and G.S. Firestein. 2013. Duality of fibroblast-like synoviocytes in RA: Passive responders and imprinted aggressors. Nature Reviews Rheumatology 9 (1): 24-33. https://doi.org/ 10.1038/nrrheum.2012.190.

5. Okamoto, H., K. Shidara, D. Hoshi, and N. Kamatani. 2007. Anti-arthritis effects of vitamin $\mathrm{K}(2)$ (menaquinone-4)-A new potential therapeutic strategy for rheumatoid arthritis. The FEBS Journal 274 (17): 4588-4594. https://doi.org/10.1111/ j.1742-4658.2007.05987.x.

6. Huber, L.C., O. Distler, I. Tarner, R.E. Gay, S. Gay, and T. Pap. 2006. Synovial fibroblasts: Key players in rheumatoid arthritis. Rheumatology (Oxford) 45 (6): 669-675. https://doi.org/10.1093/ rheumatology/kel065.

7. Montecucco, F., and F. Mach. 2009. Common inflammatory mediators orchestrate pathophysiological processes in rheumatoid arthritis and atherosclerosis. Rheumatology (Oxford) 48 (1): 11-22. https://doi.org/10.1093/rheumatology/ken395.

8. Mendell, J.T., and E.N. Olson. 2012. MicroRNAs in stress signaling and human disease. Cell 148 (6): 1172-1187. https://doi.org/ 10.1016/j.cell.2012.02.005.

9. Filkova, M., B. Aradi, L. Senolt, C. Ospelt, S. Vettori, H. Mann, A. Filer, et al. 2014. Association of circulating miR-223 and miR-16 with disease activity in patients with early rheumatoid arthritis. Annals of the Rheumatic Diseases 73 (10): 1898-1904. https:// doi.org/10.1136/annrheumdis-2012-202815.

10. Zhu, S., W. Pan, X. Song, Y. Liu, X. Shao, Y. Tang, D. Liang, et al. 2012. The microRNA miR-23b suppresses IL-17-associated autoimmune inflammation by targeting TAB2, TAB3 and IKK-alpha. Nature Medicine 18 (7): 1077-1086. https://doi.org/10.1038/ nm.2815.

11. Sun, Y., Q. Li, H. Gui, D.P. Xu, Y.L. Yang, D.F. Su, and X. Liu. 2013. MicroRNA-124 mediates the cholinergic anti-inflammatory action through inhibiting the production of pro-inflammatory cytokines. Cell Research 23 (11): 1270-1283. https://doi.org/10.1038/ cr.2013.116.

12. Philippe, L., G. Alsaleh, A. Pichot, E. Ostermann, G. Zuber, B. Frisch, J. Sibilia, S. Pfeffer, S. Bahram, D. Wachsmann, and P.
Georgel. 2013. MiR-20a regulates ASK1 expression and TLR4dependent cytokine release in rheumatoid fibroblast-like synoviocytes. Annals of the Rheumatic Diseases 72 (6): 10711079. https://doi.org/10.1136/annrheumdis-2012-201654.

13. Liu, D., N. Zhang, J. Zhang, H. Zhao, and X. Wang. 2016. miR-410 suppresses the expression of interleukin- 6 as well as renal fibrosis in the pathogenesis of lupus nephritis. Clinical and Experimental Pharmacology \& Physiology 43 (6): 616-625. https://doi.org/ 10.1111/1440-1681.12576.

14. Wang, J., H. Ye, D. Zhang, K. Cheng, Y. Hu, X. Yu, L. Lu, J. Hu, C. Zuo, B. Qian, Y. Yu, S. Liu, G. Liu, C. Mao, and S. Liu. 2017. Cancer-derived circulating microRNAs promote tumor angiogenesis by entering dendritic cells to degrade highly complementary microRNAs. Theranostics 7 (6): 1407-1421. https://doi.org/ 10.7150/thno. 18262.

15. Ke, X., Y. Yuan, C. Guo, Y. Yang, Q. Pu, X. Hu, K. Tang, et al. 2017. MiR-410 induces stemness by inhibiting Gsk3beta but upregulating beta-catenin in non-small cells lung cancer. Oncotarget 8 (7): 4 11371. https://doi.org/10.18632/oncotarget.14529.

16. Liu, D., N. Zhang, X. Zhang, M. Qin, Y. Dong, and L. Jin. 2016. MiR-410 down-regulates the expression of interleukin-10 by targeting STAT3 in the pathogenesis of systemic lupus erythematosus. Cellular Physiology and Biochemistry 39 (1): 303-315. https:// doi.org/10.1159/000445625.

17. Arnett, F.C., S.M. Edworthy, D.A. Bloch, D.J. McShane, J.F. Fries, N.S. Cooper, L.A. Healey, et al. 1988. The American Rheumatism Association 1987 revised criteria for the classification of rheumatoid arthritis. Arthritis and Rheumatism 31 (3): 315-324.

18. Zhang, Y., D. Zhang, J. Lv, S. Wang, and Q. Zhang. 2018. miR-410$3 \mathrm{p}$ promotes prostate cancer progression via regulating PTEN/AKT/ mTOR signaling pathway. Biochemical and Biophysical Research Communications 503: 2459-2465. https://doi.org/10.1016/ j.bbrc.2018.06.176.

19. Bartok, B., and G.S. Firestein. 2010. Fibroblast-like synoviocytes: Key effector cells in rheumatoid arthritis. Immunological Reviews 233 (1): 233-255. https://doi.org/ 10.1111/j.0105-2896.2009.00859.x.

20. Dasuri, K., M. Antonovici, K. Chen, K. Wong, K. Standing, W. Ens, H. El-Gabalawy, and J.A. Wilkins. 2004. The synovial proteome: Analysis of fibroblast-like synoviocytes. Arthritis Research \& Therapy 6 (2): R161-R168. https://doi.org/10.1186/ar1153.

21. Kokkonen, H., I. Soderstrom, J. Rocklov, G. Hallmans, K. Lejon, and S. Rantapaa Dahlqvist. 2010. Up-regulation of cytokines and chemokines predates the onset of rheumatoid arthritis. Arthritis and Rheumatism 62 (2): 383-391. https://doi.org/10.1002/art.27186.

22. Feldmann, M., E. Andreakos, C. Smith, J. Bondeson, S. Yoshimura, S. Kiriakidis, C. Monaco, et al. 2002. Is NF-kappaB a useful therapeutic target in rheumatoid arthritis? Ann Rheum Dis 61 (Suppl 2): ii13-ii18.

23. Li, D., Y. Yang, G. Zhu, X. Liu, M. Zhao, X. Li, and Q. Yang. 2015. MicroRNA-410 promotes cell proliferation by targeting BRD7 in non-small cell lung cancer. FEBS Letters 589 (17): 2218-2223. https://doi.org/10.1016/j.febslet.2015.06.031.

24. Dong, L., Y. Pu, L. Zhang, Q. Qi, L. Xu, W. Li, C. Wei, X. Wang, S. Zhou, J. Zhu, X. Wang, F. Liu, X. Chen, and C. Su. 2018. Human umbilical cord mesenchymal stem cell-derived extracellular vesicles promote lung adenocarcinoma growth by transferring miR-410. Cell Death \& Disease 9 (2): 218. https://doi.org/10.1038/s41419-0180323-5.

25. Wu, H., J. Li, E. Guo, S. Luo, and G. Wang. 2018. MiR-410 acts as a tumor suppressor in estrogen receptor-positive breast cancer cells by directly targeting ERLIN2 via the ERS pathway. Cellular 
Physiology and Biochemistry 48 (2): 461-474. https://doi.org/ 10.1159/000491777.

26. Zhang, X., X. Ke, Q. Pu, Y. Yuan, W. Yang, X. Luo, Q. Jiang, et al. 2016. MicroRNA-410 acts as oncogene in NSCLC through downregulating SLC34A2 via activating Wnt/beta-catenin pathway. Oncotarget 7 (12): 14569-14585. https://doi.org/10.18632/ oncotarget.7538.

27. Wang, Y., J. Fu, M. Jiang, X. Zhang, L. Cheng, X. Xu, Z. Fan, J. Zhang, Q. Ye, and H. Song. 2014. MiR-410 is overexpressed in liver and colorectal tumors and enhances tumor cell growth by silencing FHL1 via a direct/indirect mechanism. PLoS One 9 (10): e108708. https://doi.org/10.1371/journal.pone.0108708.

28. Liu, C., A. Zhang, L. Cheng, and Y. Gao. 2016. miR410 regulates apoptosis by targeting Bak1 in human colorectal cancer cells. Molecular Medicine Reports 14 (1): 467-473. https://doi.org/10.3892/ mmr.2016.5271.

29. Zhang, Y.F., Y. Yu, W.Z. Song, R.M. Zhang, S. Jin, J.W. Bai, H.B. Kang, X. Wang, and X.C. Cao. 2016. miR-410-3p suppresses breast cancer progression by targeting Snail. Oncology Reports 36 (1): 480-486. https://doi.org/10.3892/or.2016.4828.

30. Guo, R., J. Gu, Z. Zhang, Y. Wang, and C. Gu. 2015. MicroRNA410 functions as a tumor suppressor by targeting angiotensin II type
1 receptor in pancreatic cancer. IUBMB Life 67 (1): 42-53. https:// doi.org/10.1002/iub.1342.

31. Zhang, Y., X. Huang, and Y. Yuan. 2017. MicroRNA-410 promotes chondrogenic differentiation of human bone marrow mesenchymal stem cells through down-regulating Wnt3a. American Journal of Translational Research 9 (1): 136-145.

32. Okamoto, T. 2006. NF-kappaB and rheumatic diseases. Endocrine, Metabolic \& Immune Disorders Drug Targets 6 (4): 359-372.

33. Yang, Y., L.L. Wang, Y.H. Li, X.N. Gao, Y. Liu, and L. Yu. 2012. Effect of CpG island methylation on microRNA expression in the k562 cell line. Biochemical Genetics 50 (1-2): 122-134. https:// doi.org/10.1007/s10528-011-9478-9.

34. Clark, A.L., and F.J. Naya. 2015. MicroRNAs in the myocyte enhancer factor 2 (MEF2)-regulated Gt12-Dio3 noncoding RNA locus promote cardiomyocyte proliferation by targeting the transcriptional coactivator cited2. The Journal of Biological Chemistry 290 (38): 23162-23172. https://doi.org/10.1074/jbc.M115.672659.

35. Chien, W.W., C. Domenech, R. Catallo, T. Kaddar, J.P. Magaud, G. Salles, and M. Ffrench. 2011. Cyclin-dependent kinase 1 expression is inhibited by p16(INK4a) at the post-transcriptional level through the microRNA pathway. Oncogene 30 (16): 1880-1891. https:// doi.org/10.1038/onc.2010.570. 